\title{
Análise da interpolação de parâmetros utilizados pelo IRI na tomografia da ionosfera com o GNSS
}

\author{
Analysis of the interpolation of parameters used by IRI in ionospheric tomography using GNSS
}

Fabricio dos Santos Prol

Paulo de Oliveira Camargo

\begin{abstract}
Universidade Estadual Paulista “Júlio de Mesquita Filho" - UNESP. Rua Roberto Simonsen, 305. CEP: 19060-900.
Presidente Prudente, SP, Brasil.

fabricioprol@hotmail.com, paulo@fct.unesp.br
\end{abstract}

Artigo - Submissão: 10/07/2015 - Aceito em: 14/12/2015

\begin{abstract}
RESUMO - Este trabalho apresenta um método de tomografia da ionosfera que proporciona a redução de operações e de parâmetros em comparação à convencional modelagem a partir do GNSS. O método consiste em integrar as observáveis GNSS (Global Navigation Satellite Systems) com o modelo IRI (International Reference Ionosphere) a partir da interpolação dos principais parâmetros que descrevem o perfil da ionosfera. Os resultados apresentaram coerência da interpolação em relação aos valores de referência obtidos com o IRI, ao nível de 3x10 $0^{-4}$ TECU, mostrando ser uma técnica viável para aplicações com o GNSS.
\end{abstract}

Palavras-chave: TEC, Imageamento Ionosférico, Perfil Ionosférico.

\begin{abstract}
This paper presents a method of reducing the number of operations and parameters usually employed in ionospheric tomographic modeling using GNSS (Global Navigation Satellite System). The method is based on a linear interpolation of the principal parameters that characterize the vertical profile of the ionosphere obtained with IRI (International Reference Ionosphere) and vertical functions are directly used in the tomographic model instead of empirical orthogonal functions. The results showed consistency between the interpolation method and the reference values at the level of $3 \times 10^{-4} \mathrm{TECU}$, presenting a viable technique for GNSS applications.
\end{abstract}

Keywords: TEC, Ionospheric Imaging, Ionospheric Profile.

\section{INTRODUÇÃO}

Modelos ionosféricos desempenham um importante papel em grande parte das investigações sobre o clima espacial. O estudo de parâmetros que descrevem o estado da ionosfera é, normalmente, realizado por meio de equipamentos específicos para tal, como a ionossonda e o radar de espalhamento incoerente inclinado/oblíquo (KIRCHHOFF, 1991). Os sistemas de navegação GNSS (Global Navigation Satellite System), no entanto, têm sido utilizados nos últimos anos como uma tecnologia adicional na modelagem da ionosfera; pois, aliando a propriedade dispersiva da ionosfera aos sinais transmitidos em duas, ou mais, frequências distintas, é possível determinar o conteúdo total de elétrons, ou seja, o TEC (Total Electron Content). Ademais, uma vez que a maior concentração de elétrons reside nas camadas superiores da ionosfera, os sinais GNSS, que atravessam por diversas altitudes, podem proporcionar melhor detalhamento da alta ionosfera, em comparação aos equipamentos terrestres (DAVIES, 1990).

Os modelos ionosféricos derivados a partir das observáveis GNSS descrevem a ionosfera a partir da estimativa de valores verticais de TEC (VTEC - Vertical TEC) (SCHAER; GURTNER, 1998; PROL;
CAMARGO, 2014). Porém, erros na modelagem do VTEC ocorrem, principalmente, devido à necessidade da aplicação de uma função de mapeamento e da consideração da ionosfera como uma fina camada de altitude constante (BRUNINI et al., 2011). Desta forma, há interesse no estudo de modelos tomográficos que permitem reconstruir o perfil de densidade de elétrons a partir de sinais advindos de diferentes direções/elevações e dos diversos satélites do sistema GNSS. Contudo, a modelagem tridimensional (3D) da ionosfera requer uma maior quantidade de parâmetros em comparação ao caso bidimensional (2D).

Desta forma, neste trabalho, é apresentada uma proposta de redução da quantidade de parâmetors para a modelagem $3 \mathrm{D}$ da ionosfera, em relação aos métodos convencionais.

\section{MÉTODO DE INVESTIGAÇÃO}

$\mathrm{Na}$ página da web mantida pela National Aeronautics and Space Administration (NASA), encontrase mais de vinte diferentes modelos da ionosfera (NASA, 2014). Dentre estes, destaca-se o IRI (International Reference Ionosphere), um modelo empírico global da ionosfera, baseado em diversas fontes de dados de sondas 
espaciais e terrestres. O modelo foi desenvolvido e continua sendo aprimorado pelo Committeon Space Research (COSPAR) e pela International Union of Radio Science (URSI) em conjunto com membros de várias nações, com a intenção de obter componentes que descrevem a ionosfera, tais como densidade dos elétrons e íons, temperatura, composição iônica, velocidade do plasma e TEC (BILITZA et al., 2006).

A partir do IRI, o perfil da densidade de elétrons pode ser estimado para qualquer posição geográfica. A versão mais recente, IRI-2012, descreve o perfil a partir da divisão da ionosfera em seis camadas (D, E, E-F1, F1, F2 e topside) (REINISCH et al., 2000). Os perfis são produzidos a partir de funções específicas para cada uma das camadas da parte inferior da ionosfera e a partir do NeQuick para a parte superior (topside) da ionosfera (BILITZA et al., 2011). Tais funções requerem a estimativa de parâmetros que representam a ionosfera, sendo alguns dos principais: altitude de pico da região $\mathrm{F} 2$ $\left(h_{m}\right)$, espessura $\left(B_{0}\right)$ e fator de escala $\left(B_{1}\right)$ da região $\mathrm{F} 2$, e achatamento da parte inferior da ionosfera $\left(H_{0}\right)$.

A versão mais recente do IRI descreve a região F2 da ionosfera a partir da formulação introduzida por Ramakrishnan e Rawer (1972):

$$
n_{e}=\frac{n_{m} e^{-X_{1}}}{\cosh (X)}
$$

com:

$$
X=\frac{\left(h_{m}-h\right)}{B_{0}}
$$

sendo $n_{e}$ a densidade de elétrons em uma dada altitude (h), $n_{m}$ a densidade de elétrons para o pico da altitude $h_{m}$ e cosh o cosseno hiperbólico.

De acordo com Bilitza et al. (2006), o NeQuick é o modelo utilizado para descrever a parte superior da ionosfera, onde a densidade de elétrons é derivada a partir da função de Epstein:

$$
n_{e}=\frac{4 n_{m} e^{z}}{\left[1+e^{z}\right]^{2}}
$$

onde:

$$
z=\frac{\left(h-h_{m}\right)}{H_{s}}
$$

O achatamento do perfil $\left(H_{s}\right)$ é descrito a partir de um fator de escala que varia com a altitude:

$$
H_{s}=H_{0}\left[1+\frac{12,5\left(h-h_{m}\right)}{100 H_{0}+0,125\left(h-h_{m}\right)}\right]
$$

O fator de escala $\left(H_{0}=k B_{b o t}\right)$ é definido em função do parâmetro de achatamento da parte inferior da ionosfera $\left(B_{b o t}\right)$ e de um fator de correção $(k)$.

Com base nos perfis obtidos pelo IRI, as informações verticais são, habitualmente, utilizadas para a construção de EOFs (Empirical Orthogonal Functions) a partir da modelagem de parâmetros que definem uma superfície. A equação de observação da modelagem da superfície ionosférica tridimensional é dada por (HOWE et al., 1998):

$$
\begin{gathered}
\text { TEC }=\sum_{k=1}^{k_{\max }} \sum_{n=0}^{n_{\max }} \sum_{m=0}^{n} \\
a_{k n m} \int_{r}^{s}\left\{E_{k}(h) \tilde{P}_{n m}\left(\sin \left(\varphi_{m}^{i p}\right)\right) \cos \left(m \lambda^{s}\right)\right\} d s+ \\
b_{k n m} \int_{r}^{s}\left\{E_{k}(h) \tilde{P}_{n m}\left(\sin \left(\varphi_{m}^{i p}\right)\right) \sin \left(m \lambda^{s}\right)\right\} d s
\end{gathered}
$$

onde $\varphi_{m}^{i p}$ é a latitude geomagnética no ponto ionosférico IPP (Ionospheric Pierce Point), $\lambda^{s}$ é a longitude do IPP fixo à longitude do Sol medida no tempo universal (UT Universal Time), $n_{\max }$ é o máximo grau da expansão em série, $a$ e $b$ são os coeficientes dos harmônicos esféricos a serem estimados e $P_{n m}(x)$ representa os polinômios associados de Legendre normalizados de grau $n$ e ordem $m$. O termo $E_{k}(h)$ é o valor contido no vetor $E_{k}$, correspondente a atitude $h$, para cada um dos $k$ modos das EOFs.

A decomposição em valores singulares (SVD Singular Value Decomposition) permite estimar os coeficientes dos harmônicos esféricos (MITCHELL; SPENCER, 2003). Porém, é possível aplicar o Método dos Mínimos Quadrados (MMQ) diretamente, sendo necessário inserir injunções nas EOFs, dadas por seus autovalores. O sistema de equação possui $\left[\left(n_{\max }+\right.\right.$ $\left.1)^{2}\right] * k_{\max }$ parâmetros incógnitos, onde a integral da função é representada por uma soma finita de TEC determinado para diversos IPPs em diferentes altitudes.

Neste trabalho, ao invés das EOFs, propõe-se reduzir o número de parâmetros da modelagem $3 \mathrm{D}$ a partir da utilização direta das funções do IRI. Desta forma, a equação de observação que representa os harmônicos é dada por:

$$
\begin{gathered}
\text { TEC }=\sum_{n=0}^{n_{\max }} \sum_{m=0}^{n} \\
a_{k n m} \int_{r}^{s}\left\{f(h) \tilde{P}_{n m}\left(\sin \left(\varphi_{m}^{i p}\right)\right) \cos \left(m \lambda^{s}\right)\right\} d s+ \\
b_{k n m} \int_{r}^{s}\left\{f(h) \tilde{P}_{n m}\left(\sin \left(\varphi_{m}^{i p}\right)\right) \sin \left(m \lambda^{s}\right)\right\} d s
\end{gathered}
$$

na qual $f(h)$ representa o modelo vertical que descreve a ionosfera a partir do IRI. Mantendo, portanto, o sistema de equação com $\left(n_{\max }+1\right)^{2}$ parâmetros incógnitos. Nota-se que a equação (7) possui uma menor quantidade de parâmetros a serem estimados em comparação com a equação (6), no entanto, as variações temporais da função serão adequadas às funções do IRI, o que requer $o$ processamento do IRI para cada instante em que as 
observáveis serão utilizadas para mapear a ionosfera (p. ex., intervalo de observação de 15 segundos). Como alternativa, pode-se determinar os principais parâmetros do IRI a cada hora e interpolá-los para cada instante de observação do GNSS. No entanto, com uso da equação (7), as observações de TEC irão contribuir apenas na estimativa do pico da densidade de elétrons. Por outro lado, ao utilizar as EOFs, estas poderão ser assumidas proporcionais a alguma função vertical, de forma que o TEC contribuirá na estimativa da altitude de pico e do fator de escala, além do pico da densidade de elétrons. Porém, vale ressaltar que, mesmo utilizando de EOFs, a estimativa dos parâmetros que descrevem a variabilidade vertical da ionosfera é comprometida devido à geometria limitada do GNSS para a reconstrução tomográfica. Uma simulação da limitação da geometria do GNSS para a reconstrução tomográfica é apresentada por Prol (2015).

\section{RESULTADOS E DISCUSSÕES}

Uma análise da interpolação dos principais parâmetros do IRI é apresentada nas Figuras 1 a 4 . Os parâmetros do IRI $\left(\mathrm{h}_{\mathrm{m}}, \mathrm{B} 0, \mathrm{~B} 1, \mathrm{H} 0\right)$ são obtidos a partir do IRI-2012 para 01:00 UT e 02:00 UT do dia 01/01/2013. O IRI possui diversas opções de configuração para realizar o cálculo dos parâmetros, sendo que neste experimento foi utilizada a opção BIL-2000 (BILITZA et al., 2000) no cálculo de B0 e B1. Utilizou-se o código fonte do modelo disponível pelo IRI (IRI, 2014) para o cálculo dos parâmetros. Os mapas globais foram produzidos, de forma que as discrepâncias da interpolação foram calculadas para 01:30 UT.

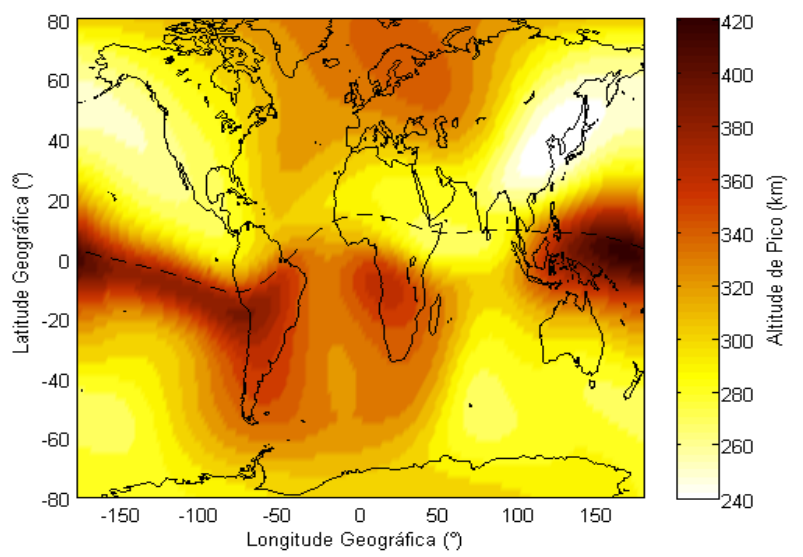

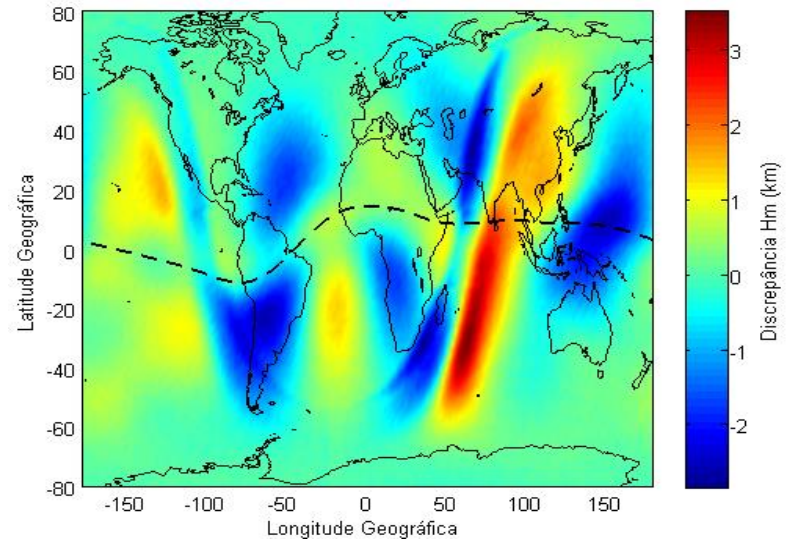

Figura 1 - Variação de $h_{m}$ e discrepância da interpolação.
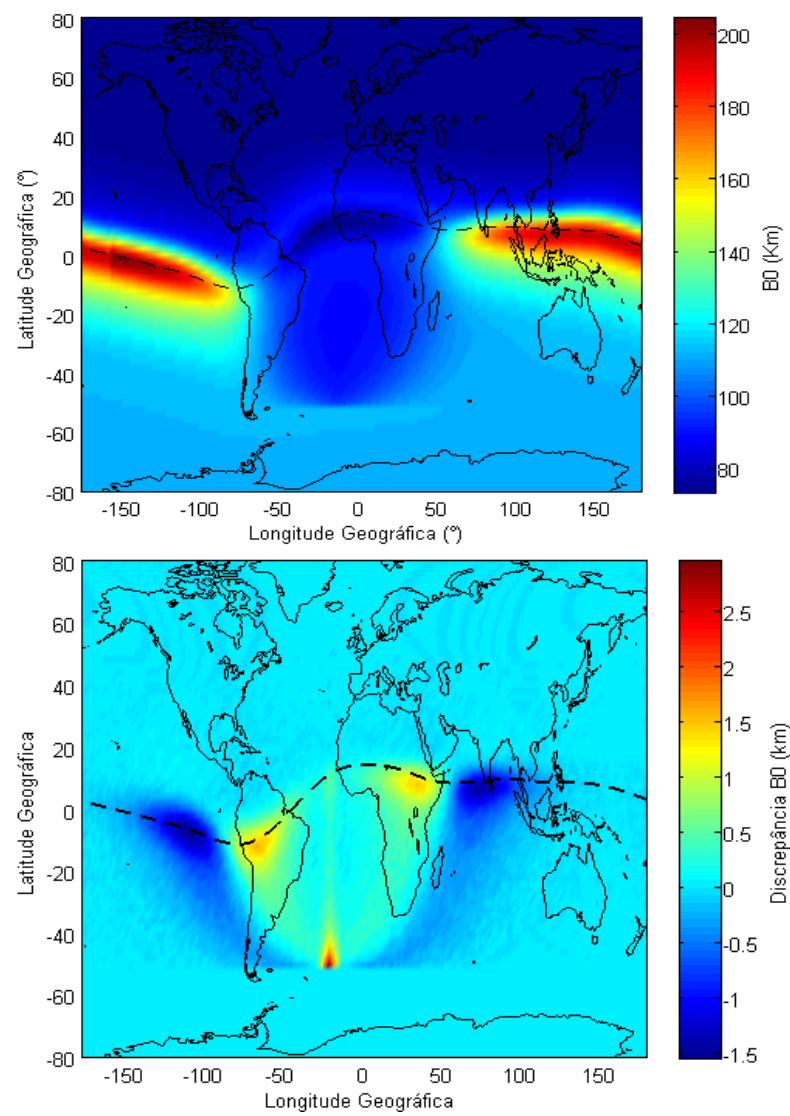

Figura 2 - Magnitude do parâmetro B0 e discrepância da interpolação (BIL-2000). 

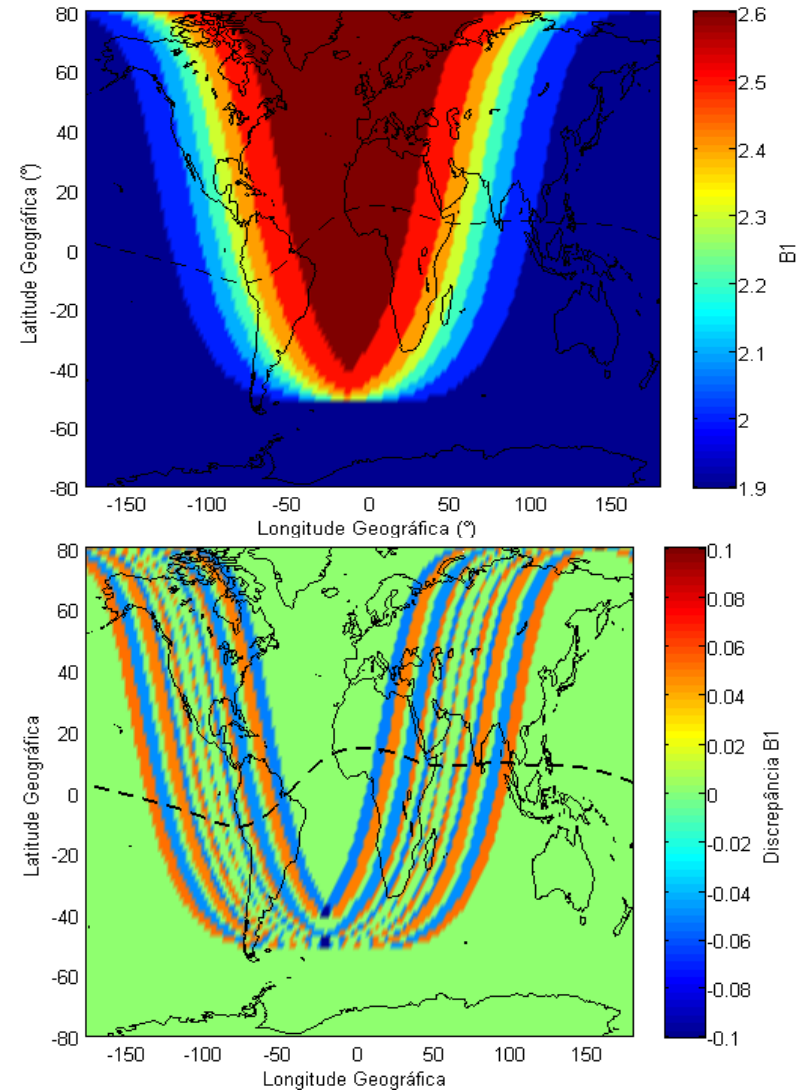

Figura 3 - Magnitude do parâmetro B1 e discrepância da interpolação (BIL-2000).
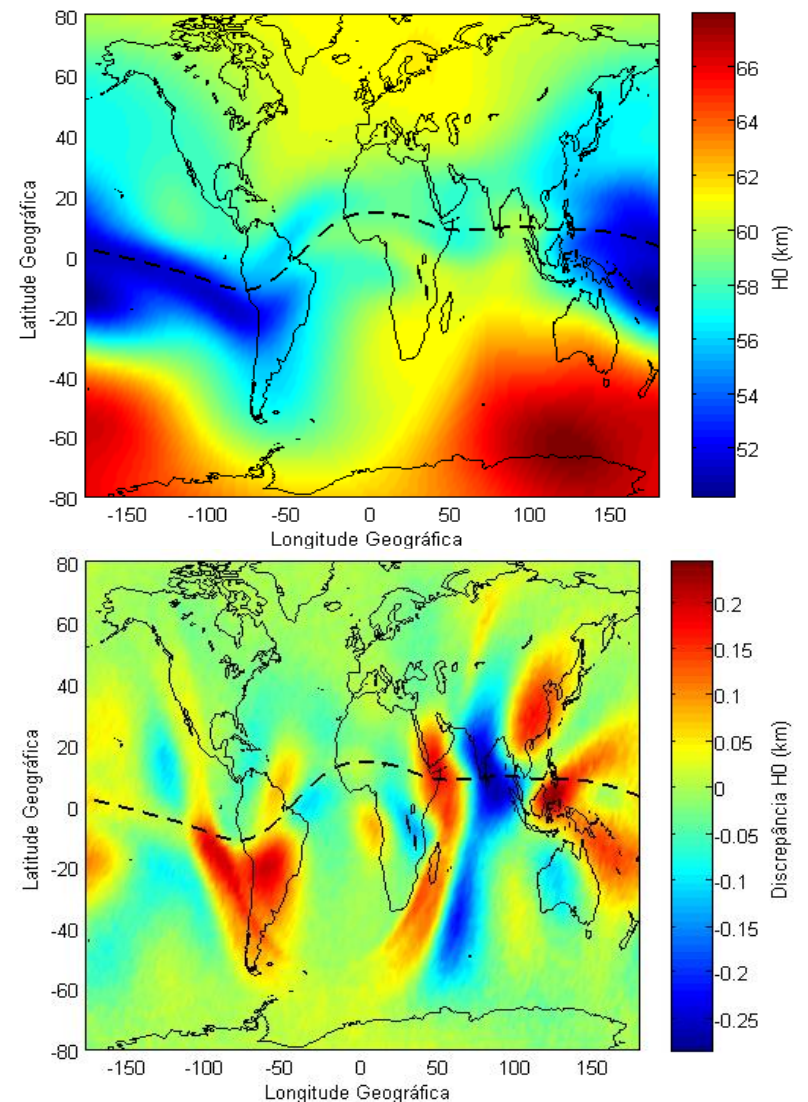

Figura 4 - Magnitude do parâmetro H0 e discrepância da interpolação.
Nota-se que com uso da interpolação, todos os parâmetros necessários para reconstruir o perfil a partir das equações utilizadas no IRI são determinados com valores relativamente inferiores à sua respectiva magnitude. Além disso, há uma grande quantidade de posições em que a interpolação proporciona uma discrepância que tende a zero.

A fim de verificar o impacto das discrepâncias nos parâmetros, a figura 5 apresenta os perfis construídos com a interpolação e com os valores calculados com o IRI. Determinou-se a posição geográfica $10^{\circ} \mathrm{N}$ e $50^{\circ} \mathrm{E}$, a qual apresentou significativas discrepâncias em todos os parâmetros: $\Delta \mathrm{h}_{\mathrm{m}}=0,8 \mathrm{~km}, \Delta \mathrm{B} 0=0,4 \mathrm{~km}, \Delta \mathrm{B} 1=0,1 \quad$ e $\Delta \mathrm{H} 0=0,2 \mathrm{~km}$.

Verifica-se, a partir do método de interpolação linear dos parâmetros, que o perfil calculado representa aproximadamente o perfil de referência, pois a diferença entre o TEC calculado e o interpolado é da ordem de $3 \times 10^{-4}$ unidades de TEC (TECU - TEC Units). Desta forma, o processo de interpolação pode ser considerado viável na prática, ainda mais, pois a estimativa do TEC será realizada a partir das observações do GNSS e as funções verticais irão apenas descrever o perfil.

\section{CONCLUSÃO}

Os métodos convencionais para realizar a tomografia tridimensional da ionosfera a partir de dados GNSS, são baseados na construção de Funções Ortogonais Empíricas. No entanto, o processo de ajustamento requer a estimativa de uma maior quantidade de coeficientes harmônicos em comparação ao caso bidimensional. Um método baseado na interpolação dos principais parâmetros das funções verticais do IRI foi apresentado, em que o número de parâmetros a ser estimado é igual ao modelo bidimensional. No método proposto, as observações de TEC contribuem na estimativa do pico da densidade eletrônica, enquanto que o uso de EOFs possibilita que as observações de TEC sejam utilizadas na estimativa da altura de pico e do fator de escala, além do pico da densidade eletrônica.

No experimento, verificou-se que as diferenças entre os valores de referência e os interpolados, para os parâmetros necessários na reconstrução dos perfis, atingiram um máximo de $1,5 \times 10^{9} \mathrm{el} / \mathrm{m}^{3}$. Mostrando ser um método viável para a construção de perfis utilizando o TEC a partir das observáveis GNSS; pois o experimento realizado proporcionou um erro de $3 \times 10^{-4}$ TECU, para uma posição em que todos os parâmetros apresentam os maiores erros na interpolação. 

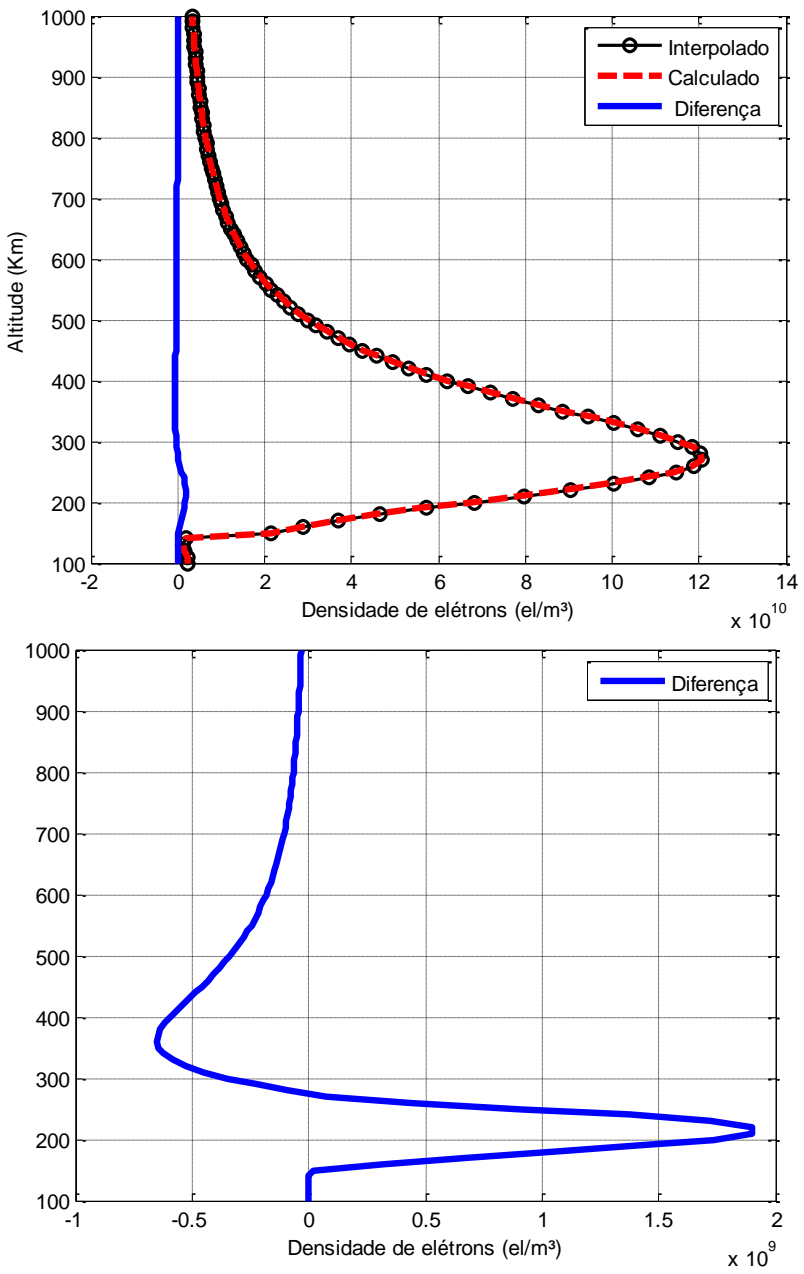

Figura 5 - Diferença visual e quantitativa da interpolação para a posição $50^{\circ} \mathrm{E}$ e $10^{\circ} \mathrm{N}$.

\section{AGRADECIMENTOS}

Os autores agradecem a bolsa de Mestrado da CAPES (Coordenação de Aperfeiçoamento de Pessoal de Nível Superior) e ao CNPq (Conselho Nacional de Desenvolvimento Científico e Tecnológico) pela bolsa de Produtividade em Pesquisa (processo n ${ }^{\circ}$ 309924/2013-8).

\section{REFERÊNCIAS BIBLIOGRÁFICAS}

BILITZA, D.; RADICELLA, S.; REINISCH，B. W.; ADENIYI, J.; MOSERT, M.; ZHANG, S.; OBROU, O. New B0 and B1 models for IRI. Advances in Space Research, v. 25, p. 89-95, 2000.

BILITZA, D.; REINISCH, B. W.; RADICELLA, S. M.; PULINETS, S.; GULYAEVA, T.; TRISKOVA, L. Improvements of the International Reference Ionosphere model for the topside electron density profile. Radio Science, v. 41, n. 5, 2006.

BILITZA, D.; MCKINNEL, L., A.; REINISCH, B.; ROWELL, T. F. The international reference ionosphere today and in the future. J. Geod, v. 85, p. 909-920, 2011.

BRUNINI, C.; CAMILION, E.; AZPILICUETA, F. Simulation study of the influence of the ionospheric layer height in the thin layer ionospheric model. $J$. Geod, v. 85, p. 637-645, 2011.

DAVIES, K. Ionospheric Radio. London: Peter Peregrinus Ltd., 580 p, 1990.

HOWE, B. M.; RUNCIMAN, K.; SECAN, J. A. Tomography of the ionosphere: Four-dimensional simulations. Radio Science, v. 33, n. 1, pp. 109-128, 1998.

IRI, International Reference Ionosphere. Disponível em: <iri.gsfc.nasa.gov/>. Acesso em junho de 2014.

KIRCHHOFF, W. J. H. Introdução à Geofísica Espacial. 1. ed. São Paulo: Nova Stella, 1991. 149 p.

MITCHELL, C. N.; SPENCER, P. S. A threedimensional time-dependent algorithm for ionospheric imaging using GPS. Annals of Geophysics. v. 46, p. 687-697, 2003.

NASA, National Aeronautics and Space Administration. Ionospheric Modeling. Disponível em: <http://ccmc.gsfc.nasa.gov/modelweb/>. Acesso em junho de 2014.

PROL, F. S.; CAMARGO, P. O. Estimativa da tendência diferencial do código nos receptores GNSS. Boletim de Ciências Geodésicas, v. 20, n. 4, p. 735-749, 2014.

PROL, F. S. Tomografia da ionosfera a partir do GNSS e técnicas algébricas: estudo de caso para a região brasileira. 2015. Dissertação (Mestrado em Ciências Cartográficas) - Universidade Estadual Paulista, Presidente Prudente.

RAMAKRISHNAN, S.; K. RAWER. Model electron density profiles obtained by empirical procedures. In: Space Research XII, Akademie-Verlag, Berlin, p. 1253-1261, 1972.

REINISCH, B. W.; HUANG, X. Redefining the IRI F1 layer profile. Advances in Space Research, v. 25, p. 81-88, 2000.

SCHAER, S; GURTNER, W. IONEX: The IONosphere Map Exchange Format Version 1. Proceedings of the IGS AC Workshop, Darmstadt, Germany, 1998. 